\title{
Estrategias de gestión y comunicación de los líderes intermedios en la aplicación de los ECTS ${ }^{1}$
}

\author{
Helena Troiano \\ Josep Maria Masjuan \\ Marina Elias \\ Universitat Autònoma de Barcelona. Grup de Recerca sobre Educació i Treball (GRET) \\ helena.troiano@uab.cat; josepmaria.masjuan@uab.cat; marina.elias@uab.cat
}

Aceptado: 09-06-2010

\section{Resumen}

Los datos que se utilizan en este artículo proceden de una investigación sobre el Proceso de Bolonia llevada a cabo en diez titulaciones de una sola universidad; seis de ellas forman parte del proyecto piloto para incorporar los European Credit Transfer System (ECTS). En este trabajo, nos centramos en cómo los líderes intermedios de las titulaciones piloto estudiadas ponen en marcha la implementación de los ECTS siguiendo estrategias diferenciadas de gestión, generación de consenso y comunicación. Asimismo, se examinan las respuestas que el profesorado de las mismas titulaciones da, en términos de valoración de la gestión, las actitudes ante la reforma y el grado de información percibido. El análisis nos permite identificar algunas oportunidades y constricciones — derivadas de las formas de gestión consideradas legítimas por los miembros de esta institución universitaria- ante las que se sitúa la acción de los líderes intermedios.

Palabras clave: gestión universitaria; líderes intermedios; Espacio Europeo de Educación Superior (EEES); reforma universitaria; European Credit Transfer System (ECTS).

Abstract. Middle Managers' Communication and Management Strategies During the Implementation of the European Credit Transfer System

This paper uses data obtained from a research project about the Bologna Process, carried out in ten degree programmes at one university; six of these degrees are part of a pilot project to implement the European Credit Transfer System (ECTS). In this article, we examine how middle managers in these pilot degrees employ various strategies of management, consensus generation and communication while carrying out the implementation of ECTS. In addition, we examine the responses from academics pertaining to management

1. Esta investigación forma parte del Plan Nacional de Investigación Científica, Desarrollo e Investigación Tecnológica (BSO2003-06395/CPSO) y ha sido financiada por el Ministerio de Educación y Ciencia y el FEDER. 
evaluation, attitudes regarding the reform, and the perceived amount of information. This analysis allows us to identify a number of opportunities and constrictions - derived from management forms considered legitimate by the members of this university - that middle managers have to take into account.

Key words: higher education management; middle management; European Higher Education Area (EHEA); university reform; European Credit Transfer System (ECTS).

\begin{aligned} & \multicolumn{2}{c}{ Sumario } \\ & Introducción Metodología \\ & La gestión universitaria: de la colegialidad La valoración de los líderes \\ & al «nuevo gerencialismo» Estrategias de comunicación y consenso \\ & El contexto específico Discusión de resultados \\ & de la universidad española Conclusiones \\ & Modelo de gestión, comunicación Referencias bibliográficas \\ & y liderazgo como factor de éxito \end{aligned}

\section{Introducción}

La incorporación de la universidad española al Espacio Europeo de Educación Superior (EEES) es el reto actual en el cual culminan toda una serie de cambios que han afectado al sistema universitario durante las últimas décadas. En efecto, nos encontramos ante una transformación larga que afecta a cuestiones relacionadas con la estructura de los estudios, con asuntos metodológicos, demográficos, sociales, pero también con la forma de gestión de las instituciones universitarias. Algunos autores (Deem, 1998; Trowler, 1998a) han denominado a esta última forma "nuevo gerencialismo» —aunque quizá ya no podamos considerarlo tan nuevo-, lo cual denota la aplicación de técnicas empresariales a la gestión de organizaciones que tradicionalmente se han alejado de estas técnicas, como las entidades sin ánimo de lucro o las instituciones públicas.

Aunque es posible hallar algunos antecedentes, un buen hito a considerar como primer impulso de la reforma universitaria en España es la LRU (Ley de Reforma Universitaria, LRU, 1983 - Partido Socialista). Dicha ley pretendía conseguir una respuesta más sensible de la universidad a las demandas del mercado laboral. Así, se intentó profesionalizar más los títulos, pero, al mismo tiempo, se quiso potenciar la investigación, de manera que se modificó la organización de la universidad con el fin de lograr estos dos objetivos; todo ello acompañado con medidas de democratización de la gestión.

El despliegue de la LRU ha sido largo, ya que incluía la reforma de los planes de estudio de todas las titulaciones universitarias, de manera que no es hasta la Ley Orgánica de Universidades (LOU, 2001 —Partido Popular) que se introducen nuevos cambios en la gestión de las universidades y las reformas necesarias para la incorporación de España al EEES. Pero con la LOU que- 
dan algunos temas pendientes de concreción que, finalmente, una nueva ley (Ley Orgánica 4/2007, de 12 de abril —Partido Socialista) se ha encargado de zanjar. En el caso que nos ocupa, el de la gestión universitaria, lo cual ha incrementado la autonomía de las universidades.

Mientras tanto, algunas universidades españolas han iniciado procesos piloto de incorporación de algunas de las propuestas europeas, siempre con la limitación de mantenerse en la normativa de los planes de estudio vigentes. Este mismo es el caso de Cataluña, donde, aprovechando el marco de la Llei d'Universitats de Catalunya (LLUC, 2003), el Departamento de Universidades y Sociedad de la Información (DURSI) inició, en 2004, un proyecto piloto para promover la adaptación de algunas titulaciones al EEES.

El objetivo de este artículo es exponer un estudio de caso en una universidad catalana que cuenta con titulaciones piloto desde el primer año en que se pusieron en marcha. El análisis gira en torno a las formas de toma de decisiones y estrategias seguidas por los líderes intermedios durante el desarrollo y la implementación de la reforma; también se atiende a las reacciones que tales formas han suscitado entre el profesorado implicado en el proceso.

En el siguiente apartado, se presentan algunos aspectos teóricos relacionados con el tema de la gestión universitaria, junto con algunas cuestiones de contexto importantes en relación con este tema. En segundo lugar, resumimos brevemente las principales conclusiones que un informe de la European University Association (2005) sobre los factores que promueven el éxito de la incorporación de la reforma europea en la educación superior señala en relación con la cuestión del «modelo de gestión y liderazgo». En tercer lugar, se expondrán algunos aspectos metodológicos del estudio. A continuación, se ofrecen, en dos bloques, los resultados obtenidos acerca de diversas cuestiones relacionadas con las estrategias de gestión y de comunicación de líderes situados en diversos niveles de la institución. Y, por último, se discuten los resultados obtenidos y se extraen las principales conclusiones.

\section{La gestión universitaria: de la colegialidad al «nuevo gerencialismo»}

La nueva forma de gestión que afecta a la universidad es común a la reestructuración de la financiación, el gobierno y la organización de los servicios públicos en casi todos los países occidentales. Se trata, como ya se ha dicho, de la legitimación y la aplicación de técnicas, valores y prácticas propios del sector privado a la gestión de organizaciones que se dedican a la provisión de servicios públicos (Deem y otros, 2007; Harris, 2007).

La introducción de esta forma gerencial ajena hasta ahora al ámbito de la organización pública se ha producido mediante fuertes críticas al poder profesional y a la supuesta mala calidad de los servicios públicos, pero también se vincula al intento de reducir el gasto público y, en el contexto de las universidades, coincide con un fuerte incremento de la cantidad de estudiantes (Deem, 1998, 2001). Se da también por supuesto que la puesta en marcha de estas nuevas estrategias y procesos llevarán a la universidad a lograr un más 
eficaz rendimiento, capaz de responder a las expectativas de los agentes externos (Neave, 2002).

El nuevo modelo de gestión entra en la universidad por diversas vías y se concreta en ejemplos variados que afectan a diferentes niveles y ámbitos de la organización. Además, la tradición universitaria de cada país desempeña un papel destacado y las nuevas formas de gestión tendrán que superponerse a las instituciones propias, lo cual dará lugar a combinaciones específicas muy diversas según cada contexto (Vaira, 2004).

Algunos ejemplos en los que es posible encontrar trazas de la introducción de este nuevo modelo de gestión son: la puesta en marcha de formas competitivas para el acceso a recursos, la implementación de agencias externas de evaluación, la creciente consolidación de la figura del gestor permanente (Santiago et al., 2006; Deem y Brehony, 2005), la reestructuración de los planes de estudio en créditos y módulos que fragmentan y hacen más vendible el conocimiento (Trowler, 1998a; Becher, 1994), la participación de agentes externos a la universidad en sus órganos de decisión y un largo etcétera (Enders y van Vught, 2007).

Una buena síntesis de estos cambios se concreta en la tipología que resume el denominado Informe Bricall (2000), basándose en modelos ya clásicos

Tabla 1. Espacio de posibilidades para el gobierno de las universidades

\begin{tabular}{|c|c|c|c|}
\hline & Burocrático & Colegial & $\begin{array}{l}\text { Empresarial } \\
\text { [gerencialista] }\end{array}$ \\
\hline Financiación & $\begin{array}{l}\text { Exclusivamente } \\
\text { pública. }\end{array}$ & $\begin{array}{l}\text { Pública o privada } \\
\text { no lucrativa. }\end{array}$ & Privada. \\
\hline Directivos & Nombrados. & Electos. & Contratados. \\
\hline $\begin{array}{l}\text { Programas } \\
\text { de enseñanza }\end{array}$ & $\begin{array}{l}\text { Determinados } \\
\text { por el estado. }\end{array}$ & $\begin{array}{l}\text { Definidos por } \\
\text { la universidad. }\end{array}$ & $\begin{array}{l}\text { Condicionados } \\
\text { por la demanda. }\end{array}$ \\
\hline Títulos académicos & $\begin{array}{l}\text { Garantizados } \\
\text { por el estado. }\end{array}$ & $\begin{array}{l}\text { Garantizados por } \\
\text { la universidad. }\end{array}$ & $\begin{array}{l}\text { Sistemas de } \\
\text { acreditación. }\end{array}$ \\
\hline Profesorado & Funcionarios. & $\begin{array}{l}\text { Contrato académico } \\
\text { (tenure). }\end{array}$ & Contrato laboral. \\
\hline Estatuto jurídico & $\begin{array}{l}\text { Organismo de } \\
\text { la Administración } \\
\text { pública. }\end{array}$ & $\begin{array}{l}\text { Corporación } \\
\text { independiente. }\end{array}$ & $\begin{array}{l}\text { Empresa o } \\
\text { asociación privada. }\end{array}$ \\
\hline Modelo de gestión & Jerárquica. & $\begin{array}{l}\text { Democrática parti- } \\
\text { cipativa. }\end{array}$ & Profesional. \\
\hline Órganos colegiados & $\begin{array}{l}\text { Consultivos } \\
\text { y electivos. }\end{array}$ & $\begin{array}{l}\text { Ejecutivos } \\
\text { y electivos. }\end{array}$ & Sólo consultivos. \\
\hline Organización & $\begin{array}{l}\text { Rígida con } \\
\text { orientación } \\
\text { profesional. }\end{array}$ & $\begin{array}{l}\text { Rígida con } \\
\text { orientación } \\
\text { disciplinaria. }\end{array}$ & Flexible. \\
\hline
\end{tabular}

Fuente: Informe Bricall, p. 417. 
de organización educativa (De Miguel, 1978; Becher y Kogan, 1980; Van Vught, 1989; Bergquist, 1992; Morgan, 1993) y en el clásico triángulo de Clark (1983) sobre los sistemas de coordinación entre el estado, la corporación académica y el mercado. La tabla 1 sintetiza los tres modelos.

Tal como se ha sugerido anteriormente, se trata de tipos ideales que han tomado forma concreta en los diferentes países y en diferentes momentos históricos a partir de la acentuación de determinadas características o la mezcla de algunas de ellas.

La investigación internacional, especialmente en Europa, ha atendido a este fenómeno de transición de un modelo a otro. Efectivamente, el paso de la colegialidad al gerencialismo parece haberse producido en diversos ámbitos de gestión y control del trabajo académico (Deem, 1998; Henkel, 2000; Rhoades y Sporn, 2002). Pero los matices sobre esta apreciación se extienden a lo largo de toda la literatura sobre el tema. Más que un simple reemplazo, se ha dado una superposición de los dos modelos que a veces ni siquiera se ha concretado en un híbrido, sino que ha generado conflicto, resistencia y fuertes contradicciones (Trowler, 1998b; Henkel, 2000; Troiano, 2004; Musselin y Becquet, 2005; Santiago et al., 2006; Amaral y Veiga, 2009).

En el plano de los líderes intermedios, estos cambios quedan reflejados en los cambios de rol generalmente asumidos.

[...] being able to define missions, objectives and strategies; having capacity to manage financial and human resources and to assume strong management leadership, in contrast to traditional academic styles of negotiation and consensus building. In short, under the new «managerialist» pressure, the performance of academic roles based upon research reputation and to a lesser extent on teaching and scholarship appears to give way to performance based upon management capacities (Greene et al. 1996; Miller 1998; Harman 2002). (Santiago et al., 2006)

A menudo, ello les coloca en una posición de difícil equilibrio entre los valores, las normas y las expectativas de sus colegas profesores, así como los de los gestores de mayor nivel en la organización - aunque estos últimos son elegidos de entre el personal académico de la misma comunidad universitaria, con lo que también podrían ser considerados "colegas profesores». Por ello, no resulta extraño encontrar a muchos de estos líderes intermedios en situaciones contradictorias, con responsabilidad y sin poder, o bien exhortando al resto de profesorado a admitir más estudiantes sin recursos, aún posicionándose ellos mismos en contra de tales medidas (Deem, 1998; Santiago et al., 2006).

\section{El contexto específico de la universidad española}

Retomando el marco concretado por el Informe Bricall (2000), se puede decir que la tendencia general de la universidad española ha sido el paso de un modelo burocrático hacia un modelo colegial, con la permanencia de algunos 
aspectos del modelo burocrático y la incorporación, más recientemente, de otros del modelo empresarial o gerencialista ${ }^{2}$.

La universidad española ha sido gobernada históricamente siguiendo los principios del modelo burocrático de tradición francesa, regido por el derecho administrativo y por las pautas de comportamiento de las administraciones públicas. Este modelo agudizó sus características debido al centralismo y a la falta de democracia del último período autoritario.

Es por ello que no representa ninguna sorpresa que la primera ley sobre universidades del actual período democrático, la Ley de Reforma Universitaria, de 1983, quisiera cambiar profundamente la gestión de la universidad acercándola al modelo colegial, con lo cual desarrolló la autonomía universitaria, recogida en la Constitución de 1978, y la democratización interna de la universidad. De hecho, todos estos aspectos habían sido ampliamente reivindicados por los actores implicados desde el tardofranquismo. La Ley mantuvo el profesorado funcionario como el eje de la actividad docente y de investigación, pero estableció diferentes categorías de profesorado contratado. A partir de la reforma de las titulaciones derivadas de esta ley, se potenció un acercamiento al mercado laboral y la incorporación de una mayor proporción de aspectos prácticos y profesionales en las titulaciones, sobre todo en las de primer ciclo.

El mismo Informe Bricall ya apunta claramente las consecuencias más negativas que pueden derivarse del modelo colegial, las cuales han sido subrayadas en el caso de España por otros autores (Mora y Vidal, 2003), fundamentalmente en lo que se refiere a la poca eficacia del sistema de gobierno para adaptarse al cambio social rápido y al excesivo peso de los intereses corporativos de los académicos en la toma de decisiones a todos los niveles.

El paso siguiente se da ya a principios del presente siglo, cuando la Ley Orgánica de Universidades de 2003 (LOU) revisa algunos aspectos de la LRU a fin de intentar aproximar la universidad española al modelo gerencialista, cambiando los sistemas de elección de cargos, restringiendo la participación en los órganos colegiales, ampliando la presencia de la sociedad civil en el órgano máximo de gobierno institucional (Consejo de Gobierno) y aumentando el peso y la categoría del profesorado contratado, pero imponiendo contrariamente un proceso más centralizado de acceso a las plazas del profesorado funcionario. $\mathrm{Al}$ mismo tiempo, normaliza la incorporación de las universidades al EEES.

De todas formas, una modificación reciente de la Ley Orgánica de Universidades (2007) ha transformado, en la dirección de la autonomía universitaria, los sistemas de acceso a las plazas de profesorado y la capacidad de decisión de las universidades en la elaboración de los planes de estudio.

Por otro lado, en Cataluña se promulgó la Llei d'Universitats de Catalunya (2003), donde se adaptaban las normativas estatales a las particularidades de

2. Siempre cabe mantenerse en la referencia a un modelo débil de gerencialismo, ya que, en comparación con otros países en los que los gestores de mayor nivel de la organización son gerentes, en España ya se ha señalado que tales gestores son elegidos entre los académicos de la propia universidad. 
la comunidad autónoma. La ley catalana apuesta decididamente por la existencia de un $50 \%$ de profesorado contratado, refuerza la mejora de la calidad y la acreditación vinculada con la Agencia Catalana de Calidad y se propone impulsar resueltamente el proceso de convergencia europea.

En este contexto, el Departamento de Universidades y Sociedad de la Información (DURSI) inició, en el año 2004, un proyecto piloto para promover la adaptación de algunas titulaciones al EEES. Así, se incorporaron inicialmente a la prueba piloto en Cataluña 22 titulaciones de diplomatura e ingeniería técnica y 25 titulaciones de licenciatura e ingeniería superior ${ }^{3}$.

Las titulaciones que se incorporaron voluntariamente al plan piloto tuvieron que realizar diferentes trabajos de adaptación durante el primer trimestre del curso 2004-2005: ajustar los planes de estudio vigentes a las propuestas europeas sin introducir cambios que vulnerasen la normativa estatal, definir los perfiles de las titulaciones y las competencias para ajustarse al espíritu profesionalizador de la reforma y preparar nuevas metodologías docentes compatibles con las nuevas orientaciones didácticas y organizativas (ECTS).

En este marco se sitúa la universidad objeto de estudio como participante en la prueba piloto mencionada. Se trata de una universidad con un número elevado de alumnos y bien situada en términos de investigación.

\section{Modelo de gestión, comunicación y liderazgo como factor de éxito}

En el informe de la European University Association (EUA, 2005), se aborda explícitamente la cuestión del modelo de gestión y comunicación que los líderes han desarrollado y se identifican algunas características de tales modelos como claves para favorecer la realización del cambio previsto ${ }^{4}$.

En concreto, este factor de éxito queda expuesto de la siguiente manera:

Factor de éxito 3: intensa comunicación intrainstitucional y equipos de gobierno firmes pero abiertos al diálogo. (EUA, 2005)

La necesidad de lideraje y de un determinado estilo de ejercerlo es un aspecto destacado en múltiples investigaciones sobre la implementación de cambios en la universidad. En algunas investigaciones actuales sobre estos temas, se destacan los límites de los líderes profesionales que aplican técnicas propias del modelo gerencialista descuidando las particularidades de la universidad e

3. Las diplomaturas y las ingenierías técnicas constan en la actualidad de tres años de duración, la mayoría de licenciaturas son de cuatro años y las ingenierías superiores y algunas licenciaturas, de cinco.

4. En tres artículos anteriores, se trataron los restantes factores de éxito del mismo informe de la EUA, junto con algunos otros aspectos complementarios (Troiano y Elias, 2006). Por un lado (Masjuan y otros, 2007), los tres aspectos más relacionados con la política universitaria: la relación con otras reformas de enseñanza superior, el equilibrio correcto entre regulación y coordinación de ámbito estatal y autonomía institucional y el apoyo financiero estatal. Y, por otro lado, el factor de encaje entre las políticas de nivel más alto y más bajo: directivas descendentes como oportunidad para las reformas ascendentes (Troiano y otros, 2010). 
incluso de los contextos disciplinarios específicos (Cryer y Elton, 1990; Deem, 1998; Trowler, 1998a; Martin, 1999; Troiano, 2004).

Es en este sentido que las especificidades institucionales desempeñan un papel muy relevante en la adecuación del modelo de gestión adoptado. El informe europeo que tomamos como referencia inicia el apartado en estos términos:

Es interesante el hecho de que muchas instituciones observaron que el proceso de reforma de Bolonia había servido como una oportunidad especial para el fortalecimiento de la coherencia institucional, el fomento de la transparencia y la coordinación institucional, así como el refuerzo de los canales de comunicación horizontales. (EUA, 2005)

Pero también parece evidente que la asunción de responsabilidad y dirección por parte de los líderes se convierte en un extremo ineludible. Las características que recogen como constantes en las instituciones exitosas son las siguientes (EUA, 2005):

- Un miembro del equipo de liderazgo institucional asume la responsabilidad del proceso, destacando aquellas instituciones con un liderazgo proactivo.

- Existencia de un grupo de coordinación a nivel de facultad, en el cual el decano o algún vicedecano ha desempeñado un papel fundamental de coordinación.

- En algunas instituciones, los sindicatos de estudiantes y sus líderes han facilitado las tareas de implementación.

- El liderazgo en todos los niveles requiere capacidad de diálogo genuino y, al mismo tiempo, capacidad de proponer unas líneas globalizadoras, especialmente al inicio del proceso.

En definitiva, y tal y como explicita el informe:

El desafío continúa siendo la instauración, por parte del lideraje, de un marco de gestión de alto nivel, equilibrado con el espacio para las iniciativas ascendentes en el seno de departamentos y facultades. (EUA, 2005)

\section{Metodología}

Los datos que utilizamos en este artículo proceden de una investigación de mayor alcance que se plantea como un estudio de casos ${ }^{5}$. Aunque en la investigación original se estudiaron un mayor número de titulaciones de una misma universidad, para este artículo hemos utilizado solamente los datos procedentes de seis de ellas, que son las que entraron inicialmente a formar parte del plan piloto de incorporación a los créditos europeos (Humanidades, Relaciones Laborales, Sociología, Matemáticas, Física e Informática).

5. En la dirección http://www.uab.es/gret se puede encontrar el informe general de este trabajo, en el cual se concretan las características técnicas de la investigación. 
Tal como se indicó anteriormente, el plan piloto se ha iniciado sin reformar la estructura de los planes de estudio, pendiente de cambios legislativos, por consiguiente, se limitó a la incorporación de los perfiles profesionales de competencias y a la implantación de los ECTS, vinculada al desarrollo de una nueva metodología docente.

Las técnicas de recogida de datos incluyen entrevistas en profundidad a por lo menos tres responsables institucionales de cada titulación (habitualmente: decano, director de departamento y coordinador de titulación), citados como líderes a partir de este momento. En estas entrevistas, se trataban temas de contexto, visión sobre el mismo profesorado, proceso de reforma desde posiciones privilegiadas y algunas valoraciones sobre el proceso. También se realizaron encuestas por cuestionario con preguntas abiertas y cerradas al profesorado implicado en la titulación (su totalidad o un mínimo de cincuenta cuestionarios) y análisis de otros tipos de documentación (evaluaciones, boletines de presentación de la titulación, actas de reuniones, páginas web, etc.).

El procedimiento seguido para analizar las cuestiones que hemos seleccionado incluye el uso de varias de las fuentes descritas. En primer lugar, recogemos las referencias que los líderes hacen a los temas que nos ocupan, con lo cual damos voz a las preocupaciones y a las definiciones de la realidad que realizan los propios actores.

Sobre algunos de los temas analizados, resulta posible contrastar esta definición de la realidad que efectúan los líderes con datos procedentes de la encuesta al profesorado. En este caso, el objetivo es indagar en las posibles consecuencias que, sobre la percepción y la opinión del profesorado, pueden haber tenido determinadas estrategias emprendidas por parte de los líderes.

Es cierto que la combinación de técnicas diversas que resulta de este procedimiento complica la base metodológica de la comparación, pero, como contrapartida, ofrece una mayor riqueza de matices y nos aproxima mejor a la complejidad de un proceso de cambio como el que abordamos en la universidad actual.

\section{La valoración de los líderes}

Se comienza esta exposición de resultados por lo que podría ser considerado como consecuencia del proceso: la valoración que el profesorado emite sobre la gestión que han llevado a cabo sus líderes. En el próximo apartado, se describirán las estrategias de comunicación y gestión de los líderes que habrían contribuido a generar los presentes resultados.

En primer lugar, se hace necesario clarificar los niveles de liderazgo que han sido sometidos a valoración. Aunque, enmarcada en un estudio de caso, la investigación se ceñía a una sola universidad, la diversidad de niveles, competencias y tamaño de las diferentes entidades en la organización complicó en gran manera la decisión sobre qué niveles resultaba pertinente investigar. 
La voluntad de captar el funcionamiento real en la toma de decisiones y la puesta en marcha de la reforma llevó a adaptar la decisión sobre qué personas se debían entrevistar a la estructura organizativa existente. Y esto significa diferentes niveles de líderes intermedios para diferentes titulaciones:

- Coordinador o coordinadora de titulación. En todas las ocasiones, se entrevistó a este responsable institucional, en la mayoría de los casos, persona encargada directamente de llevar a cabo el proceso de diseño e implementación del proyecto de reforma.

- Responsable específico del proyecto piloto. En algunas titulaciones, se habían encargado a una persona o a un cargo específico las tareas de diseño e implementación del proyecto de reforma.

- Jefe del departamento mayoritario. Aunque, en la estructura universitaria actual, los departamentos no tienen responsabilidad directa sobre la organización de los grados, a menudo constituyen una estructura que aglutina personas que sienten que pertenecen a él y que difunden maneras de trabajar y pensar comunes. Es, por tanto, una entidad a tener en cuenta para entender la orientación que adoptan determinados procesos, especialmente los de reforma. En alguna ocasión, además, el departamento mayoritario era prácticamente único en la titulación.

- Decano o decana (o vicedecano o vicedecana). Si la facultad era pequeña (incluía tres titulaciones o menos), se entrevistaba directamente a su decano o, si éste lo recomendaba, al vicedecano que se hubiera hecho responsable de la realización del proyecto piloto. En el caso de facultades mayores, con más de tres titulaciones en su seno, se procuraba abarcar tanto el nivel de decanato como el de jefe de sección, para acercar más el foco de estudio al nivel de organización general de docencia en la titulación pertinente.

Así pues, para cada titulación, los líderes intermedios se sitúan en diferentes niveles de responsabilidad y de cargos. Es por ello que las preguntas de valoración de la gestión de tales líderes merecen una pequeña explicación. En primer lugar, para el ámbito general de la universidad, se interpreta que todo el profesorado se está refiriendo a los mismos líderes (rector, vicerrectores o vicerrectoras pertinentes y apoyo técnico) cuando se les pregunta « ¿Considera que el proceso de implementación de los ECTS se está haciendo adecuadamente en la Universidad?». En cambio, se estima que responden de forma contextual a la pregunta "iConsidera que el proceso de implementación de los ECTS se está haciendo adecuadamente en su titulación?». Tal formulación permite diferenciar con cierta coherencia entre la valoración que el profesorado emite sobre líderes que se sitúan más lejos en la escala de gestión o los más cercanos a su propia posición, es decir, los líderes intermedios.

Teniendo esto en cuenta, ya se puede afirmar, tal y como se observa en la tabla 2, que, entre el profesorado de las titulaciones piloto que responde a esta pregunta valorando la gestión de sus líderes, un porcentaje mucho más elevado considera siempre - excepto en el caso de Humanidades - que los 
intermedios han realizado una implementación más adecuada que los líderes de la universidad.

Una información complementaria que se obtiene a partir de las encuestas realizadas tiende a confirmar esta visión poco positiva de la gestión de los líderes más lejanos. Así, de los 322 profesores que responden a la pregunta, un $61 \%$ considera que la implementación de los ECTS va a generar conflictos entre las autoridades académicas y el profesorado. En la pregunta abierta que sigue a ésta, se recogen algunas pistas de los motivos que consideran como posibles causantes de futuros conflictos. En primer lugar, el conjunto de problemas derivados de la poca disponibilidad de recursos, tanto estrictamente económicos como humanos; ello se traduce en el incremento de trabajo junto con la extensión de un cierto sentimiento de que «El rectorado no nos apoya». En segundo lugar, otra serie de dificultades derivadas, precisamente, de la lejanía en que se sitúan los líderes de la universidad, lo cual se traduce en la percepción de que «No saben qué pasa en esta titulación específica» o "No conocen las particularidades de nuestra disciplina», "Nos imponen formas pedagógicas que no se adaptan a nuestras necesidades», «No hay comunicación real ni tampoco confianza». En último término, encontramos un grupo de argumentos que señalan al mismo profesorado como generador de conflictos como consecuencia de sus propios intereses: el profesorado mantiene mucha inercia en la docencia porque tiene la cabeza (y el corazón) centrada en la investigación.

En el apartado siguiente, se retomará la valoración de los líderes propios, que, como puede observarse en esta tabla, resulta muy dispar, puesto que abarca desde el tercio de Física hasta la totalidad de Matemáticas. Se juzga positivamente a sus líderes cercanos.

Tabla 2. Valoración de la gestión de la reforma en dos niveles de liderazgo

\begin{tabular}{lcccc}
\hline & $\begin{array}{l}\text { Valoración de los líderes } \\
\text { de la titulación }\end{array}$ & & \multicolumn{2}{l}{$\begin{array}{l}\text { Valoración de los líderes } \\
\text { de la universidad }\end{array}$} \\
\cline { 2 - 3 } \cline { 5 - 6 } $\begin{array}{l}\text { Porcentaje de: } \\
\text { valoración positiva } \\
\text { y muy positiva }\end{array}$ & $\mathrm{N}$ & $\begin{array}{l}\text { lorcentaje de: se hace } \\
\text { Pastante } \text { muy bien }\end{array}$ & $\mathrm{N}$ \\
\hline Humanidades & 45,4 & 22 & 40 & 25 \\
Relaciones Laborales & 13,3 & 15 & 43,8 & 16 \\
Sociología & 26 & 23 & 75 & 28 \\
Matemáticas & 27,8 & 18 & 100 & 28 \\
Física & 16,7 & 18 & 31,8 & 22 \\
Informática & 40,7 & 27 & 65,7 & 32 \\
Total & 30,1 & 123 & 62,3 & 151 \\
\hline
\end{tabular}

Nota: respuestas posibles: 1. Nada. 2. Poco. 3. Bastante. 4. Mucho. 9. No lo sé.

Fuente: elaboración propia. 


\section{Estrategias de comunicación y consenso}

En este apartado, se ofrece un relato esquemático de las estrategias de comunicación y consenso que han seguido los líderes intermedios en las titulaciones estudiadas, así como una explicación de los resultados que obtienen en diversos ámbitos.

A efectos de claridad en la exposición, se han agrupado las titulaciones piloto en tres conjuntos según los resultados que obtienen. Los datos se exponen para los tres grupos suponiendo que hay una gradación en tales resultados, pero se han tenido en cuenta diversas variables a la hora de clasificar las titulaciones. Así pues, Matemáticas, Sociología e Informática pertenecerían al primer grupo, donde se obtienen las mejores valoraciones para sus líderes propios y en las que no se detectan problemas de información. En peores condiciones que las anteriores se sitúa la titulación de Relaciones Laborales, única que forma parte del segundo grupo, el cual cae en una situación intermedia. Las dos últimas titulaciones, Humanidades y Física, dejan entrever algunos puntos débiles en relación con la gestión y el liderazgo que hacen prever algunos problemas de implementación.

En el primer grupo, las titulaciones de Sociología y de Matemáticas siguen estrategias esencialmente similares, ya que basan la gestión del cambio en asegurar la comunicación y la generación de consenso mediante el uso de los canales y los espacios propios del departamento mayoritario en cada caso. Se trata de departamentos muy centrales a las dos titulaciones y que históricamente se habían implicado activamente en cuestiones docentes, por ejemplo: organizando seminarios sobre docencia. Por ello no resulta tan extraño que los líderes de ambas titulaciones pretendan llevar a cabo un cambio importante contando con el apoyo de los departamentos respectivos. En su discurso, se toma en cuenta y se explicita el papel informal que éstos desempeñan en las cuestiones relacionadas con la docencia.

En ambos casos, el de Matemáticas y Sociología, y reforzando los hallazgos del informe europeo que sirve de referencia a este trabajo (EUA, 2005), se ve como hay algún líder de la titulación (decano, vicedecano o coordinador de titulación) que asume personalmente la responsabilidad de activar el cambio, en conjunción - y consolidación - con el establecimiento de consenso con los líderes respectivos de los departamentos mayoritarios.

La estrategia descrita para estas dos primeras titulaciones, Matemáticas y Sociología, tiene en común con la de Informática el hecho de contar con una persona que asume como propia la responsabilidad de llevar a cabo la reforma y también el haber puesto en marcha estrategias múltiples de comunicación y consenso. En el caso de la titulación de Informática, a las varias reuniones informativas dirigidas a todo el profesorado, se añaden la elaboración de una página web abierta a contribuciones (wiki) con el fin de difundir información realmente relevante y mantener al profesorado informado de todas las decisiones que se van tomando en las comisiones respectivas.

Los resultados que se obtienen en las tres titulaciones expuestas hasta ahora —Matemáticas, Sociología e Informática— son similares en cuanto a algunas 
cuestiones. Básicamente, resulta que los esfuerzos de difusión de la información dan sus frutos y nos situamos ante las tres titulaciones con el profesorado mejor informado sobre el proceso de reforma (entre el 71 y el $86 \%$ bastante o muy informado sobre los ECTS).

También habíamos visto en el apartado anterior que las tres titulaciones - Matemáticas, Sociología e Informática - se sitúan muy bien en cuanto a la valoración que su profesorado hace de cómo se está llevando a cabo la reforma en su titulación. En esta valoración indirecta de la gestión de sus líderes intermedios, Informática consigue que dos tercios respondan que la implementación se está haciendo bastante o muy bien, para Sociología son tres cuartas partes del profesorado las que realizan esta valoración y en Matemáticas resulta ser el $100 \%$.

Otro indicio para la valoración de los resultados de las fases iniciales de implementación se obtiene de una tipología construida combinando dos variables procedentes del cuestionario al profesorado (tabla 4). Por un lado, se le preguntaba si pensaba que sus prácticas docentes iban a cambiar en los próximos cinco años y, por otro, se le pedía que valorara las nuevas formas docentes que se proponía realizar con la reforma. Los tipos resultantes son los siguientes: Resistencia negativa $=$ «Mi forma de hacer docencia no cambiará en los próximos cinco años" $\mathrm{y}$ "Valoro de forma negativa los cambios docentes propuestos»; Cambio forzado $=$ «Mi forma de hacer docencia sí cambiará en los próximos cinco años» $\mathrm{y}$ "Valoro de forma negativa los cambios docentes propuestos»; Resistencia positiva $=$ «Mi forma de hacer docencia no cambiará en los próximos cinco años» y «Valoro de forma positiva los cambios docentes propuestos»; Cambio positivo $=$ "Mi forma de hacer docencia sí cambiará en los próximos cinco años» $\mathrm{y}$ «Valoro de forma positiva los cambios docentes propuestos».

Si nos centramos en las actitudes sobre el cambio que muestran el conjunto de profesores de Matemáticas, Sociología e Informática, vemos que los resultados son más dispares (tabla 4). Sociología presenta una mayoría de profesores (44\%) de cambio positivo. Informática obtiene el mismo porcentaje (44\%) de

Tabla 3. Grado de información sobre los ECTS declarado por el profesorado.

\begin{tabular}{lcc}
\hline & \multicolumn{2}{c}{ Grado de información respecto de los ECTS } \\
\cline { 2 - 3 } & Porcentaje de: bastante y muy informado & N \\
\hline Humanidades & 54,7 & 42 \\
Relaciones Laborales & 53,1 & 32 \\
Sociología & 86,1 & 36 \\
Matemáticas & 71,1 & 38 \\
Física & 52,5 & 40 \\
Informática & 78 & 41 \\
Total & 5,9 & 229 \\
\hline
\end{tabular}

Fuente: elaboración propia. 
profesores del tipo de cambio positivo, pero también se observa otra concentración en las respuestas de su profesorado: un tercio piensa que tal cambio se va a producir, pero lo valora de forma negativa (cambio forzado).

En las entrevistas a los líderes de la titulación de Informática, más de una vez surgió la apreciación de que el nivel de consenso obtenido al comienzo del proceso parecía estar deteriorándose. Así, cada vez se escuchan más voces críticas con la medida de haberse avanzado. Se opina que el proceso de reforma es demasiado incierto y está comportando demasiado esfuerzo, quizá hubiera sido más prudente esperar a ver cómo se resolvían muchas incógnitas del contexto legal y político. Podría ser que esta evolución del discurso que rompe el consenso inicialmente logrado fuera el que explicara la polarización en las expectativas que muestra el profesorado de Informática, reflejo de un malestar que los líderes ya han percibido.

Por último, en la titulación de Matemáticas, la situación respecto a la tipología de actitudes (tabla 4) es bastante peor, ya que la mayoría $(45,2 \%)$ se posiciona en resistencia negativa. Una información complementaria es que la apreciación de que va a generarse conflicto entre el profesorado y las autoridades académicas de la universidad es muy alta en esta titulación. Los líderes destacan en las entrevistas este extremo cuando señalan la percepción de poco apoyo que reciben de instancias superiores el profesorado y ellos mismos. Asimismo, se difunde cierto escepticismo sobre los métodos docentes en base a los resultados mediocres obtenidos en reformas anteriores. Este escepticismo, sumado a la percepción de mala gestión en niveles superiores a la titulación, podría desembocar en la concentración de profesorado en la categoría que hemos visto - no cambio en cinco años y mala valoración de la docencia propuesta.

La titulación de Relaciones Laborales sigue unas estrategias ciertamente diferenciadas de los tres casos anteriores. Se les parece, sin embargo, en el hecho de que también cuenta con un líder que asume como proyecto propio la realización del proyecto piloto de reforma. Los canales de comunicación y

Tabla 4. Tipología de actitudes sobre el futuro: expectativas y valoración del cambio futuro del profesorado

\begin{tabular}{lrrccr}
\hline & $\begin{array}{c}\text { Resistencia } \\
\text { negativa }\end{array}$ & $\begin{array}{c}\text { Cambio } \\
\text { forzado }\end{array}$ & $\begin{array}{c}\text { Resistencia } \\
\text { positiva }\end{array}$ & $\begin{array}{c}\text { Cambio } \\
\text { positivo }\end{array}$ & N \\
\hline Humanidades & 23,3 & 36,7 & 10 & 30 & $100 \%(30)$ \\
Relaciones Laborales & 10,5 & 21,1 & 16 & 53 & $100 \%(19)$ \\
Sociología & 18,8 & 25 & 13 & 44 & $100 \%(32)$ \\
Matemáticas & 45,2 & 12,9 & 26 & 16 & $100 \%(31)$ \\
Física & 16 & 12 & 12 & 60 & $100 \%(25)$ \\
Informática & 14,7 & 32,4 & 8,8 & 44 & $100 \%(34)$ \\
Total & 22,2 & 24 & 14 & 39,8 & $100 \%(171)$ \\
\hline
\end{tabular}

Fuente: elaboración propia. 
consenso que se utilizan son los propios de la titulación (no del departamento, lo cual sería inviable, porque se trata de una titulación en la que confluyen un gran número de departamentos), esto es, comisiones específicas de docencia y de reforma.

En este sentido, la forma de gestión resulta más similar a la de Informática, pero la difusión de las decisiones, la obtención de consenso, el diagnóstico del estado de opinión, etc. son tareas que se llevan a cabo de manera informal, focalizada en las personas implicadas y a través de una relación cara a cara.

Los resultados que se obtienen en Relaciones Laborales son peores en relación con las cuestiones referidas a la gestión: el profesorado se muestra menos informado que en los tres casos anteriores (53\% bastante y mucho), y no se valora tan bien la implementación de la reforma en la propia titulación (44\% bastante y muy adecuadamente). No obstante, si atendemos a las actitudes sobre el futuro de su profesorado (tabla 4), resulta que los que muestran actitudes de cambio positivo superan la mitad de los docentes.

Los líderes de la titulación de Relaciones Laborales son conscientes de esta disposición positiva a la innovación en docencia y es por ello que consideran viable la implementación de la reforma de manera poco traumática. En cambio, sólo algunos de los líderes son capaces de identificar problemas de información (sobreinformación de mensajes poco relevantes o difusión desigual de la información importante) o de toma de decisiones (contextos en que se toman decisiones específicas muy desvinculadas de los centros de poder y/o muy focalizadas entre profesorado afectado), problemas que no son muy ajenos a los sistemas de comunicación y consenso que, como éste, confían en las redes informales de la organización.

Las dos últimas titulaciones piloto, Humanidades y Física, presentan de formas diferentes algún tipo de inconveniente relacionado con el liderazgo. Ambas titulaciones pertenecen a «macrofacultades» que incluyen muchas otras titulaciones. Esto dificulta, por simple distancia, que un líder a nivel del decanato pueda asumir de manera eficiente la responsabilidad personal de hacer avanzar la reforma. $\mathrm{Y}$ en ambos casos alguna persona de los respectivos decanatos desempeñaron un papel fundamental en la decisión (o incitación) a participar en el plan piloto de la reforma. En un nivel más próximo a la titulación, nadie toma el relevo con suficiente convencimiento. En el caso de Física, la incertidumbre sobre el futuro — qué decisiones se deberán tomar, qué modelo se deberá seguir - se apodera de la titulación, no sólo del profesorado, sino también de los propios líderes. Y el caso de Humanidades es peor: los líderes sienten que se están tomando decisiones sin contar con ellos, se consideran víctimas de una imposición y viven el proceso de forma conflictiva.

Las dificultades en la generación de consenso que se derivan de esta última situación en Humanidades resultan evidentes: los líderes intermedios más próximos a la titulación no buscarán el consenso del profesorado si ellos mismos no están de acuerdo con el proceso. Igualmente, la difusión de la información ya encuentra obstáculos en el canal que circula del decanato a la titulación. 
En cambio, la situación en Física no se presenta tan conflictiva. En primer lugar, las decisiones específicas se toman en la comisión pertinente y se discuten con el profesorado afectado ${ }^{6}$. De momento, esta vía de acción no ha generado conflictos manifiestos. En segundo lugar, los líderes se han implicado en la organización de reuniones informativas abiertas para difundir la información pertinente, aunque ellos mismos cuenten que la indecisión sobre cuestiones cruciales limita la posibilidad de llevar a cabo una política y una estrategia de difusión de información clara y decidida.

En ambas titulaciones, Humanidades y Física, se plantea la intención de diseñar en el futuro campañas de información profundas y extensas.

Los niveles de información en la titulación de Física, como cabría esperar, son muy bajos para tratarse de una titulación piloto, sólo el $53 \%$ se siente bastante o muy informado. Este extremo también se pone de manifiesto en la baja respuesta que obtenemos en el cuestionario en las secciones relacionadas específicamente con los créditos europeos y su implementación. Así, contando con este descenso en el volumen de respuesta, vemos que el 60\% de los encuestados en Física pertenece al tipo de cambio positivo (tabla 4), es decir, el que valora positivamente las nuevas formas docentes y cree que en los próximos cinco años habrá cambiado las suyas. En cambio, de la mitad que responden a la valoración sobre la implementación, sólo un tercio cree que ha sido adecuada en su titulación (frente a dos tercios que creen lo mismo de entre todo el profesorado de titulaciones piloto, tabla 2).

Los propios líderes de Física perciben la existencia de grupos muy diferenciados respecto de la actitud y la vinculación con la reforma: de los más enterados, activos y entusiastas, a los muy hostiles, y con un gran grupo intermedio que muestra una actitud indiferente hacia el tema. Asimismo, las dificultades relacionadas con su propia indecisión les hace intuir que se encuentran ante un proceso muy incierto y que el profesorado efectivamente lo percibe así.

En Humanidades, el nivel de información que testimonia el profesorado es mayor (56,4\% bastante y mucho), a pesar de que los líderes habían señalado obstáculos en el proceso de difusión.

Este conjunto de profesorado relativamente bien informado no valora muy bien la nueva propuesta docente (tabla 4) y es el único que considera que la implementación se está llevando a cabo mejor en la universidad que en su propia titulación (tabla 2).

La fama que precede al profesorado de la titulación de Humanidades es la de que se dedica y es innovador en cuestiones docentes, así que su potencial pedagógico parece importante. En el mismo sentido hablan los líderes (es una de las justificaciones para elegirla como titulación piloto) y los porcentajes de innovación en la dirección de un aprendizaje más activo, efectivamente, alcanzan los dos tercios del profesorado. Sin embargo, el descontento y las valoracio-

6. Recordemos, sin embargo, que esta estrategia basada en la red informal no siempre da buenos resultados. A menudo implica la sensación de que se está prescindiendo de los canales colegiales formalmente establecidos. 
nes negativas se manifiestan una y otra vez. Parece probable que la sensación de imposición para pasar a incorporarse al plan piloto se haya extendido más allá de los líderes intermedios más próximos a la titulación y haya impregnado también al resto del profesorado.

\section{Discusión de resultados}

En este artículo, se ha atendido a las estrategias de comunicación y gestión que los líderes intermedios adoptan con el fin de poner en marcha una reforma. Ha quedado claro que las diferentes formas de proceder pueden dar lugar a resultados diversos, pero el alcance de tal hallazgo es evidentemente limitado.

En primer lugar, cabe señalar que aquí sólo se analiza la disposición en las etapas iniciales del proceso de reforma en un contexto de proyectos piloto. La gestión de un cambio de esta magnitud se extiende en el tiempo y pasa por fases que es necesario atender con mucho cuidado. Una vez comenzado el cambio, hay que mantenerlo, evitar que el profesorado que lo realiza se sienta inseguro y retroceda ante la incertidumbre. El apoyo que puedan suministrar los líderes intermedios en las instituciones para continuar adelante puede llegar a ser determinante.

En segundo lugar, en este trabajo, se presentan tan sólo algunos de los factores que se consideran relevantes para la consecución de un cambio. Está claro que confluyen en el proceso muchos otros factores, algunos de los cuales han sido tratados en otros artículos que presentan otros resultados parciales de esta misma investigación que nos ocupa. Entre éstos últimos, cabe destacar que en Masjuan y otros (2007) se ponen de manifiesto el cansancio acumulado debido a la rapidez de los cambios experimentados a raíz de ésta y otras reformas anteriores y también los problemas provocados por la distribución específica de recursos humanos, mientras que en Troiano y otros (2010) se hace hincapié en el obstáculo que supone el hecho de que las preferencias y los incentivos estén centradas de forma preeminente en la investigación, no en la docencia. Y, por otro lado, se constata la importancia que la elaboración de un proyecto de reforma propio, ajustado a la idiosincrasia de cada titulación, tiene de cara a conseguir la implicación del profesorado de la titulación, especialmente cuando ésta se encuentra originalmente lejos de la filosofía propuesta por la reforma.

Los líderes intermedios son, pues, capaces de generar el proyecto de reforma con características específicas y ajustadas a su propio contexto que el informe de la European University Association (2005) identifica como la necesaria capacidad de proponer unas líneas globalizadoras, en particular al inicio del proceso.

Pero el análisis de los datos indica que las posibilidades de acción de los líderes intermedios se extienden aún más allá, puesto que, mediante la generación de consenso y la extensión de los canales de comunicación, pueden ser capaces de concretar y legitimar el proceso de cambio, o, al contrario, deslegitimarlo y generar conflicto (o no evitarlo). 
En todo caso, y respaldando de nuevo otro de los hallazgos del informe europeo de referencia en este artículo (EUA, 2005), se constata la importancia de que haya un líder comprometido con el cambio, que se haga responsable de este cometido.

Aunque tampoco es suficiente la presencia de este líder intermedio responsable, ya que la forma de ejercicio arroja más o menos probabilidades de éxito. Lo primero que merece la pena tener en cuenta es que una iniciativa de difusión de información «razonable» en términos de frecuencia y número de profesorado al que se dirige no acostumbra a ser suficiente. Las titulaciones en que el profesorado se considera realmente informado han utilizado estrategias múltiples, frecuentes y masivas para difundir incansablemente la información pertinente.

También hemos visto que el «diálogo genuino» al que se refiere el mismo informe (EUA, 2005) desempeña un importante papel. El profesorado debe sentir que se le ha tenido en cuenta en la toma de decisiones o, por lo menos, que éstas se han tomado en los canales legítimos de decisión. Esto hace referencia a la actitud consultiva — aunque asertiva, recordemos - de la dirección, pero también al cuidado de que, en la consulta, se haya incluido a todo el profesorado, no sólo a una parte de él.

La actitud del profesorado nos sitúa en un contexto institucional donde se considera que hay formas legítimas de tomar decisiones y otras que no lo son. Así, se ha puesto de manifiesto que resulta importante que la tarea de concreción del proyecto de reforma se efectúe mediante una gestión que respete las reglas del juego legítimas en el contexto en que se realiza. Y, a pesar de las grandes tendencias en la gestión de las universidades en el mundo, a pesar de la convivencia simultánea de diversas perspectivas sobre las formas de gestión propias de la universidad, el modelo colegial es reciente y consistente en la universidad española. El profesorado puede llegar a rechazar un proyecto si no se respetan las normas de colegialidad que considera legítimas. Esto último vendría a reforzar el argumento de Santiago y otros (2006) en relación con los modelos imperantes en la universidad portuguesa y probablemente en las de muchos otros países: los nuevos modelos de gestión no siempre son un híbrido entre los modelos colegial y gerencial, sino que a menudo se constituyen en modelos llenos de contradicciones que aún se están resolviendo, con un futuro incierto.

Tal y como sintetizaba Bricall (2000), en España la forma de gestión colegial ha calado en la cultura propia de la universidad a partir del despliegue de la LRU y no es hasta tiempos más recientes que la gestión gerencial avanza de manera irregular. Este retraso relativo se enmarca en el contexto político de transición hacia la democracia, con mayor legitimidad conferida a las formas de organización colegiales, en principio, consideradas más democráticas —aunque a veces sólo aparentemente. La superposición de determinadas formas organizativas transnacionales a las tradiciones y tendencias de cada país dentro de su marco político e institucional es la tesis que defiende Vaira (2004) sobre cómo se extienden los cambios que afectan a la universidad en el mundo. 
En este contexto de predominancia del modelo colegial entre el profesorado, no resulta legítimo todo lo que los líderes a veces piensan que sí lo es, como, por ejemplo, «by-pasar» (en su propia terminología) algunos centros de poder consolidados o apoyarse en la red informal de relaciones, tomando decisiones exclusivamente con los afectados por la medida a implementar. Nos situamos ante un ejemplo concreto de lo que muchos autores hemos visto que identifican como los límites con los que se encuentran los gestores que aplican técnicas gerencialistas en contextos en que no son plenamente legítimas (Cryer y Elton, 1990; Deem, 1998; Trowler, 1998a; Martin, 1999; Troiano, 2004).

Por último, vale decir que este trabajo también ha contribuido a identificar otro punto clave en el proceso de gestión del cambio. Se ha puesto de manifiesto un cierto malestar y una sensación de conflictividad en las relaciones con los líderes superiores dentro de la misma institución universitaria. Evidentemente, el trabajo de reducir la presión, crear proyectos propios, animar, reconocer el trabajo, etc. es lógico que puedan hacerlo de forma más eficiente los líderes intermedios, que son los más próximos al profesorado. Pero para los líderes superiores hay una tarea ineludible a llevar a cabo: facilitar el trabajo de estos líderes intermedios. Cuando alguna de las instancias falla, repercute en la tensión que debe soportar la otra.

Así pues, como hemos visto que revelaban otros investigadores (Deem, 1998; Santiago et al., 2006), también en un contexto de "gerencialismo débil» como es el nuestro (o quizá todavía con más intensidad precisamente por ello), es posible detectar la contradicción en la que se ven atrapados los líderes intermedios entre la lealtad a los «colegas» académicos y a los «jefes» gestores de mayor nivel.

\section{Conclusiones}

1. Los líderes intermedios de esta institución universitaria tienen mucha capacidad de acción sobre la definición de un proyecto de reforma y el proceso a seguir para implementarlo. Por tanto, se encuentran con la posibilidad de legitimar y hacer aceptable el proyecto para el resto del profesorado.

2. Aunque el modelo de gestión gerencial haya podido entrar en algunos ámbitos del mundo universitario, el modelo prevalente entre el profesorado continúa siendo el colegial. Esto significa que rechazará cualquier proyecto de reforma que, en su proceso de definición, considere que vulnera las normas legítimas de la institución; en nuestro caso, información y diálogo extensivos a toda la comunidad de académicos.

3. Las estrategias de difusión de la información seguidas por los líderes intermedios sólo consiguen alcanzar a porcentajes mayoritarios de profesorado cuando se definen como masivas: por muchos canales diversos y reiteradas en el tiempo.

4. Las estrategias de consenso mediante la toma de decisiones seguidas por los líderes intermedios sólo consiguen ser consideradas legítimas por parte de 
la mayoría del profesorado cuando tienen en cuenta los ámbitos de toma de decisiones colegiales - - legítimos e inclusivos.

5. El profesorado percibe menor apoyo para afrontar la reforma por parte de los gestores de nivel superior que de los líderes intermedios. Es por ello que prevén mayor conflictividad en este ámbito.

\section{Referencias bibliográficas}

Amaral, A. y VeigA, A. (2009). «Survey on the implementation of the Bologna process in Portugal». Higher Education, 57 (1), 57-69.

Becher, T. (1994). "The State and the university curriculum in Britain». European Journal of Education, 29 (3), 231-246.

Becher, T. y Kogan, M. (1980). Process and Structure in Higher Education. Londres: Heinemann.

BergQuist, W.H. (1992). The Four Cultures of the Academy. San Francisco: JosseyBass.

BRICALL, J.M. (2000). Informe Universidad 2000. Barcelona: Conferencia de Rectores de las Universidades Españolas (CRUE).

Clark, B. (1983). The Higher Education System. Berkeley: University of California Press.

CRYeR, P. y Elton, L. (1990). «Catastrophe Theory: a unified model for educational change». Studies in Higher Education, 15 (1).

DeEm, R. (1998). "NNew managerialism" in higher education: The management of performances and cultures in universities». International Studies in the Sociology of Education, 8 (1), 47-70.

- (2001). «Globalisation, new managerialism, academic capitalism and entrepreneurialism in universities; is the local dimension still important?». Comparative Education, 37 (1), 7-20.

DeEm, R. y BrehonY, K.J. (2005). «Management as ideology: The case of "New Managerialism" in higher education». Oxford Review of Education, 31 (2), 213231.

Deem, R.; Hillyard, S. y Reed, M. (2007). Knowledge, higher education, and the new managerialism: The changing management of UK universities. Oxford: Oxford University Press.

ENDERS, J. y VAN VUGHT, F. (2007). Towards a cartography of higher education policy change: A Festschrift in Honour of Guy Neave. Center for Higher Education Policy Studies (CHEPS). University of Twente, the Netherlands.

EUA, European University Association (S. ReICHert \& Ch. Tauch) (2005). Tendències IV: Les universitats europees implementen Bolonya. Barcelona: Generalitat de Catalunya, DURSI.

HARRIS, S. (2007). The Governance of Education: How neo-liberalism is transforming policy and practice. Londres: Continuum Press.

HenKel, M. (2000). Academic Identities and Policy Change in Higher Education. Londres: Jessica Kingsley Publisher.

«Ley de Reforma Universitaria. Ley Orgánica 11/1983, de 25 de agosto». Boletín Oficial del Estado, 209, 1 de septiembre de 1983.

«Ley Orgánica 4/2007, de 12 de abril, por la que se modifica la Ley Orgánica 6/2001, de 21 de diciembre, de universidades». Boletín Oficial del Estado, 89, 13 de abril de 2007. 
«Ley Orgánica de universidades 6/2001, de 21 de diciembre». Boletín Oficial del Estado, 307,24 de diciembre de 2001.

«Llei 1/2003, de 19 de febrer, d'universitats de Catalunya». Diari Oficial de la Generalitat de Catalunya, 3826, 20 de febrero de 2003.

Martin, E. (1999). Changing Academic Work: Developing the Learning University. Buckingham, UK: Society for Research into Higher Education and the Open University Press.

MaSjuAn, J.M.; Troiano, H. y Elias, M. (2007). «Los factores de éxito de las universidades europeas en el proceso de incorporación al espacio europeo de educación superior y la experiencia de una universidad catalana». Educar, 40, 49-67.

Miguel, J. de (1978). Anatomía de una universidad: Para un estudio de sociología de las organizaciones. Barcelona: Dopesa.

MORA, J.G. y VIDAL, J. (2003). «Two decades of Changes in Spanish Universities: Learning the hard way». Cher 16th Annual Conference, Porto, 4 a 6 de septiembre.

Morgan, G. (1993). Imágenes de la organización. Cuernavaca, México: Ra-Ma.

Musselin, Ch. y BeCQueT, V. (2005). «Academic Work And Academic Identities: A Comparison Of Four Disciplines». Cher 18th Annual Conference. Jyväskylään, 1 a 3 de septiembre.

NEAVE, G. (2002). «The stakeholder perspective historically explored». En: ENDERS, J. y Fulton, O. (eds.). Higher Education in a Globalising World. Dordrecht: Kluwer Academic Publishers, 17-38.

RHOADES, G. y SPORN, B. (2002). «New models of management and shifting modes and costs of production: Europe and the United States». Tertiary Education and Management, 8, 3-28.

Santiago, R.; Carvalho, T.; Amaral, A. y Lynn Meek, V. (2006). «Changing patterns in the middle management of higher education institutions: The case of Portugal». Higher Education, 52, 215-250.

Troiano, H. (2004). «Modelos de toma de decisiones en la reforma de planes de estudio universitarios». Revista de Educación, 334, 235-258.

Troiano, H. y Elias, M. (2006). «Faculty Staff Facing New University Profiles». Cher 19th Annual Conference. Kassel, 7 a 9 de septiembre.

Troiano, H.; Masjuan, J.M. y Elias, M. (2010). «La recontextualización de las políticas de incorporación al Espacio Europeo de Educación Superior: Un estudio de caso». Revista de Educación, 351, 283-310.

TROWLER, P.R. (1998a). «What managerialists Forget: higher education credit frameworks and managerialist ideology». International Studies in Sociology of Education, 8 (1), 91-110.

- (1998b). Academics Responding to Change. Buckingham: SRHE \& Open University Press.

VAIRA, M. (2004). «Globalization and higher education organizational change: a framework for analysis». Higher Education, 48, 483-510.

VAN Vught, F.A. (ed.) (1989). Governmental Strategies and Innovation in Higher Education. Londres: Jessica Kingsley. 